\title{
Media Governance im interregionalen Vergleich - Informelle Regulierung in Italien und Mexiko
}

\author{
Klinger, Ulrike
}

\begin{abstract}
In modern mass democracies, the principle of freedom of the press limits the scope of state intervention in the media sector. Presumably 'soft' instruments of self- and informal regulation can be potential alternatives. But why do informal practices and institutions fail to promote media pluralism effectively? Both Italy and Mexico exhibit the highest rates of media concentration among OECD member states, both are considered defective democracies and both have a long tradition of informal regulation. The article concludes that informality does not serve as an effective regulatory mechanism of media pluralism. It obstructs the creation of transparent rules for new actors in the media sector. In addition, a lack of awareness and low professionalization in the media sector hamper the promotion of pluralism. Das Gebot der Pressefreiheit begrenzt in modernen Massendemokratien die Interventionsmöglichkeiten staatlicher Akteure im Medienbereich. Dadurch werden vermeintlich „softe“ Instrumente von Selbstregulierung und informeller Regulierung zu naheliegenden Alternativen. Doch warum gelingt es informellen Praktiken und Institutionen nicht, Medienpluralismus effektiv zu befördern? Italien und Mexiko weisen die höchste Medienkonzentration in der OECD auf, beide gelten als defekte Demokratien und informelle Regelsetzung hat in beiden Ländern Tradition. Der Beitrag kommt zum Ergebnis, dass Informalität nicht zu einer effektiven Regulierung von Medienpluralismus führt, weil sie sowohl die Schaffung transparenter Regeln für neue Akteure auf dem Medienmarkt verhindert und Pluralismusförderung am mangelnden Problembewusstsein und geringer Professionalisierung im Mediensektor scheitert.
\end{abstract}

DOI: https://doi.org/10.1007/s12286-014-0188-7

Posted at the Zurich Open Repository and Archive, University of Zurich

ZORA URL: https://doi.org/10.5167/uzh-102737

Journal Article

Published Version

Originally published at:

Klinger, Ulrike (2014). Media Governance im interregionalen Vergleich - Informelle Regulierung in Italien und Mexiko. Zeitschrift für Vergleichende Politikwissenschaft, 8(S1):35-59.

DOI: https://doi.org/10.1007/s12286-014-0188-7 


\title{
Media Governance im interregionalen Vergleich - Informelle Regulierung in Italien und Mexiko
}

\author{
Ulrike Klinger
}

\begin{abstract}
Zusammenfassung: Das Gebot der Pressefreiheit begrenzt in modernen Massendemokratien die Interventionsmöglichkeiten staatlicher Akteure im Medienbereich. Dadurch werden vermeintlich „softe“ Instrumente von Selbstregulierung und informeller Regulierung zu naheliegenden Alternativen. Doch warum gelingt es informellen Praktiken und Institutionen nicht, Medienpluralismus effektiv zu befördern? Italien und Mexiko weisen die höchste Medienkonzentration in der OECD auf, beide gelten als defekte Demokratien und informelle Regelsetzung hat in beiden Ländern Tradition. Der Beitrag kommt zum Ergebnis, dass Informalität nicht zu einer effektiven Regulierung von Medienpluralismus führt, weil sie sowohl die Schaffung transparenter Regeln für neue Akteure auf dem Medienmarkt verhindert und Pluralismusförderung am mangelnden Problembewusstsein und geringer Professionalisierung im Mediensektor scheitert.
\end{abstract}

Schlüsselwörter: Governance $\cdot$ Medien $\cdot$ Pluralismus $\cdot$ Italien $\cdot$ Mexiko

\section{Informal regulation of media governance in Italy and Mexico: an interregional comparison}

\begin{abstract}
In modern mass democracies, the principle of freedom of the press limits the scope of state intervention in the media sector. Presumably 'soft' instruments of self- and informal regulation can be potential alternatives. But why do informal practices and institutions fail to promote media pluralism effectively? Both Italy and Mexico exhibit the highest rates of media concentration among OECD member states, both are considered defective democracies and both have a long tradition of informal regulation. The article concludes that informality does not serve as an effective regulatory mechanism of media pluralism. It obstructs the creation of transparent rules for new actors in the media sector. In addition, a lack of awareness and low professionalization in the media sector hamper the promotion of pluralism.
\end{abstract}

Keywords: Governance $\cdot$ Media $\cdot$ Pluralism $\cdot$ Italy $\cdot$ Mexico

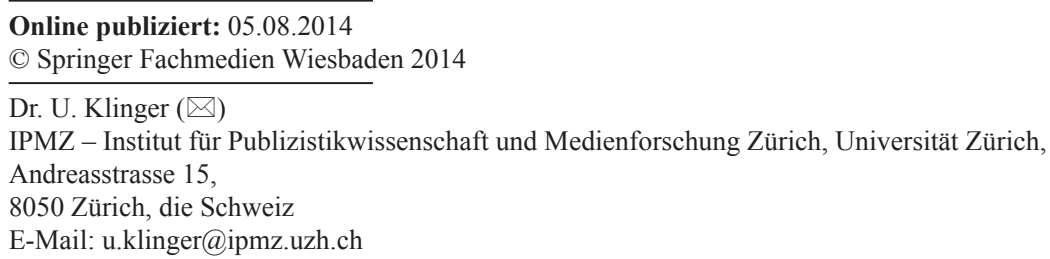




\section{Einleitung}

Wir sind hijos naturales, uneheliche, illegitime Kinder ohne Geburtsurkunde - so umschrieb ein ehemaliger Direktor des mexikanischen Senders Canal Once die Situation der staatlichen Medien, die ebenso für zivilgesellschaftliche und alternative Medien Geltung beanspruchen kann (DiBella 2005). Er meinte damit, dass aufgrund der antiquierten und defizitären Mediengesetze täglich Fernseh- und Radiostationen senden, ohne dass es für sie irgendeine rechtliche Grundlage oder formale Regulierung gibt. Und auch für Italien haben internationale Organisationen sowie das eigene Verfassungsgericht wiederholt auf eine unzureichende Kontrolle des Medienmarktes verwiesen; ein Thema, das vom Interessenkonflikt eines Ministerpräsidenten Berlusconi nur zeitweise überlagert wurde.

Es ist in der demokratietheoretischen Literatur unbestritten, dass Öffentlichkeit Pluralismus braucht; dass auf dem marketplace of ideas eine Vielzahl von Meinungen und politischen Konzepten vorhanden sein muss, weil sich auch dadurch die Qualität der Demokratie bestimmt. Gleichzeitig steht ein struktureller Pluralismus, also ein vielzähliges Angebot an Medienorganisationen, in Konflikt mit der ökonomischen Logik der Medienkonzentration - und kann daher nur ,herbeireguliert ${ }^{`}$ werden. In diesen Prozess sind zunehmend auch private Akteure eingebunden, die das engere Konzept von staatlicher Steuerung durch demokratisch legitimierte und allgemein verbindliche Gesetze und Vorgaben um neue Formen kooperativer und medieninterner Regelsetzung erweitern (wobei darauf verwiesen sein soll, dass diese Praxis wesentlich älter ist, als es die wissenschaftliche Debatte um „Governance“ nahe legt). Die zentrale Frage mit Blick auf die in westlichen Demokratien, besonders aber in Italien und Mexiko grassierende Medienkonzentration ist dabei folgende: Warum gelingt es selbstregulativen und informellen Praktiken und Institutionen nicht, eine effektive Regulierung von Medienpluralismus zu unterstützen und zu befördern? Diese Frage ist besonders in einem Umfeld relevant, dass einerseits von Marktversagen durch Monopolbildung geprägt ist und andererseits einer unzureichenden Mediengesetzgebung, die nicht einmal alle Medienakteure zu erfassen vermag. Hinzu kommt, dass Governance ein hilfreiches Konzept gerade bei der Analyse von Medienpolitik ist, weil dieses Politikfeld durch eine stark limitierte Interventionskompetenz des Staates charakterisiert ist. Staatliche Akteure können Medienorganisationen keinen inhaltlichen Pluralismus vorschreiben, weil diese durch die Garantie von Pressefreiheit vor solchen Interventionen geschützt sind. Dem Staat bleibt also allenfalls, strukturelle Vorgaben zu Medienkonzentrationsgrenzen festzuschreiben, während gerade in dieser Konstellation mediale Akteure selbst und in Kooperation mit staatlichen Akteuren gefordert sind.

In diesem Beitrag werde ich zunächst verdeutlichen, wie und warum Governance (als Media Governance) sich als fruchtbar für die Analyse von Medienpolitik erwiesen hat und vier Subtypen, darunter auch informelle Regulierung, voneinander unterscheiden. Anschließend werden die Spezifika von Medienpluralismus als Regulierungsziel herausgearbeitet und die Fallauswahl von Italien und Mexiko sowie das methodische Vorgehen begründet. In empirischen Kapiteln zeigt sich dann in Fallstudien, wie sich Media Governance in Italien und Mexiko ausprägen und welchen Beitrag Medienakteure in Selbstregulierung zu Medienpluralismus beitragen könnten bzw. welche Potentiale in der selbstverantworteten Regelsetzung vergeben werden. Auch Fälle von horizontaler und vertikaler informeller Regulierung werden diskutiert, in denen sich einmal politische 
Akteure durch informelle Absprachen des Rundfunksystems bemächtigen („lottizzazione") und andererseits staatliche Akteure durch Nichtregulierung zivilgesellschaftliche Medienakteure einer intransparenten Situation staatlicher Willkür aussetzen (radios comunitarias). In keinem der Fälle gelingt es aber, defizitäre Medienregulierung durch Einbezug privater Akteure, Selbstregulierung oder informelle Regeln zu substituieren oder effektiv zu ergänzen.

\section{Media Governance als informelle Regulierung}

Gibt man bei Google-Trends den Begriff, „Governance“ ein, sieht man, dass die Zahl der Suchanfragen dazu zwischen 2004 und 2013 kontinuierlich abgenommen hat. Dies tat der Popularität des Begriffes im wissenschaftlichen Gebrauch jedoch keinen Abbruch: Selten hat sich ein Konzept so wandlungsreich und anschlussfähig gezeigt wie „governance“, das nun auch in der Forschung zu politischer Kommunikation und Medienpolitik Einzug gehalten hat (Puppis 2010; Donges 2007; Terzis 2007). Seine Begriffskarriere begründet sich dabei nicht allein durch die Anschlussfähigkeit an verschiedene Diskurse und Disziplinen, sondern in der Möglichkeit, damit einen Wandel greif- und verstehbar machen zu können, der vor unseren Augen abläuft und doch so ganz neu nicht ist: Die zunehmende Beteiligung nicht-staatlicher und nicht demokratisch legitimierter Akteure an der Setzung und Durchsetzung verbindlicher Regeln im Prozess einer Ausweitung der klassischen Steuerung im Nationalstaat. Auch wenn nicht legitimierte und private Stakeholders schon immer Einfluss auf die Aushandlungen demokratischer Politik in der einen oder anderen Form genommen haben, ist es doch ihre immer selbstverständlichere Beteiligung an Politikherstellung und Durchsetzung, sowie die Ausdehnung von Regelsetzung in supranationale Mehrebenen-Systeme, die auf theoretischer Ebene eine Weiterentwicklung bestehender Steuerungs-Konzepte erforderten.

Dem Begriff der Governance liegt damit ein Politikverständnis zugrunde, bei dem es vor allem um kollektive Entscheidungen über Regeln und Ordnung geht (Benz und Dose 2010). Dies ist als zu eng und als begriffliche Verwässerung (Schlichte 2012, S. 11) kritisiert worden, auch weil es voraussetzt, dass Akteure, Institutionen und die Arenen ihrer Entscheidungsfindung bereits existieren. Claus Offe (2009) hat auf sprachlichkonzeptuelle Inkonsistenzen verwiesen und auch unterstrichen, dass die Ergänzung und Substituierung staatlichen Handelns durch private Akteure demokratietheoretisch hochproblematisch ist:

Such an arrangement for the nation-state would, however, have to do without any evident connection to democratic legislation as the source of ,binding decisions", nor would it allow for the legal regulation and review of procedures and outcomes. It much more resembles the political-institutional formation that Colin Crouch has described as ,post-democracy' under which economic actors enjoy the license to do as they please. (Offe 2009, S. 556)

Diese berechtigten kritischen Einwände werden die Karriere des Begriffs vermutlich nicht aufhalten, weshalb Offe auch dafür plädiert, Grenzen um Governance zu ziehen (was Governance ist, und was nicht) sowie Government als getrenntes eigenes Konzept 


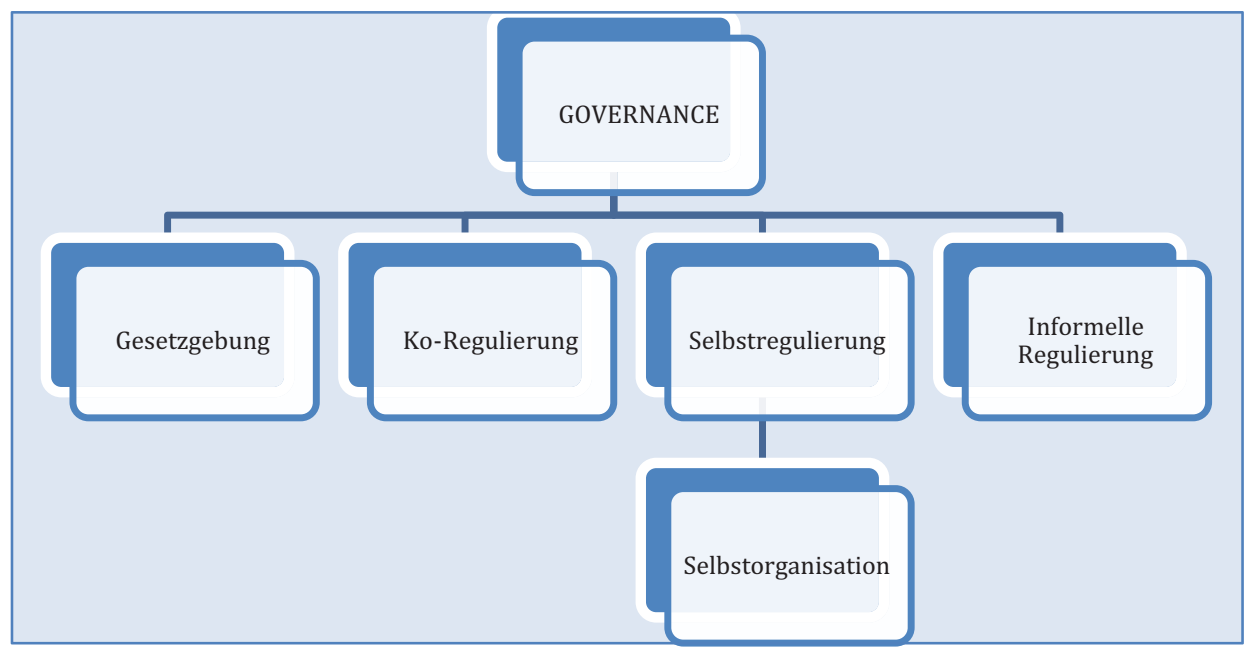

Abb. 1: Teilbereiche von Governance. (Eigene Darstellung)

zu behalten (Offe 2009, S. 552). Dies schließt an zwei grundsätzlich verschiedene Vorstellungen von staatlicher Regelsetzung an, die sich in der Governance-Debatte entwickelt haben (Puppis 2010, S. 136-137): In einem engeren Modell steht Governance für eine explizit nicht staatliche und nicht-hierarchische Regelsetzung, die als innovative Ergänzung zu Gesetzgebung und Regierungshandeln gedacht wird. In einem breiteren Modell dagegen wird Governance als übergreifender Begriff für sowohl staatliche Regulierung als auch private und zivilgesellschaftliche Regelsetzung verstanden. Dieser Beitrag schließt an diese breitere Konzeption an und versteht Mediengesetzgebung, Ko- und Selbstregulierung sowie informelle Regulierung als Teilelemente von Governance, wie in Abb. 1 dargestellt.

Der Unterschied zwischen Gesetzgebung und Ko- und Selbstregulierung liegt dabei primär auf der Akteursebene und dem Grad der Beteiligung demokratisch legitimierter Akteure an der Herstellung (allgemein) verbindlicher Regeln. Die Abgrenzung informeller Regulierung basiert hingegen auf dem Grad der formalen Institutionalisierung des Regelsetzungsprozesses, denn auch demokratisch legitimierte Akteure können an einer informalen Ordnung beteiligt sein.

Da es hier nur um informelle Regulierung, Ko- und Selbstregulierung gehen soll, wird Mediengesetzgebung als Teilelement nicht weiter diskutiert - wenngleich sie zweifellos einen wichtigen Teil von Regelsetzung im Medienbereich ausmacht und auch für Ko- und Selbstregulierung eine wesentliche Voraussetzung darstellt.

\subsection{Media Governance}

Das Konzept von Governance, verstanden als horizontale Ausweitung des klassischen Verständnisses von Regierung und Steuerung (Mayntz 2008), lässt sich besonders gut im Bereich der Medienpolitik anwenden, die von Marktversagen und einem eng begrenzten staatlichen Regulierungsspielraum geprägt ist (Jarren und Donges 2007). Medien 
haben einen Doppelcharakter als ökonomische Güter und gesellschaftliche Güter (Baker 2002), in dem sich auch die „beunruhigende Gleichstellung“ von Bürgern und Konsumenten reflektiert (Schudson 2006; Kiefer 1999). Medien als Güter unterliegen weder dem Konkurrenz- noch dem Ausschlussprinzip (non-rivalrous use; non-excludability), weil die Nutzung durch eine Person nicht die Nutzung durch eine andere Person einschränkt und einzelne Personen in der Regel nicht von der Nutzung ausgeschlossen werden können. Zudem kommt den Massenmedien die wichtige Funktion der gesellschaftlichen Selbstbeobachtung und Selbstverständigung zu. Und sie wirken nicht nur als Kommunikationsforen, sondern auch als Mobilisierungsagenten und Kontrolleure, die die gewählten Repräsentanten und Amtsinhaber beobachten und ihr Handeln öffentlich abbilden, wie es sich in den Metaphern als, watch-dogs' und des ,fourth estate' ausdrückt: „The control of the media is not like the ownership of a car industry. Media, contrary to cars or refrigerators are used to shape political opinions. The commodities produced by the media industry are highly political goods." (Fabbrini 2008, S. 202, in Anlehnung an Doris Graber). Daraus leitet sich normativ ein Regulierungsanspruch des Staates ab. Wenn zudem die Eigentumsrechte für Rundfunkfrequenzen beim Staat liegen, wird es für staatliche Akteure unmöglich, nicht zu regulieren, vielmehr wird die Ordnung des Medienmarktes zur Staatsaufgabe.

Der medienpolitische Handlungsraum von Staaten ist dabei institutionell eng begrenzt. Durch das Gebot von Pressefreiheit sind in modernen Massendemokratien zum einen die Interventionsmöglichkeiten staatlicher Akteure in den Medienbereich limitiert. Dadurch werden vermeintlich „softe“ Instrumente von Ko- und Selbstregulierung zu naheliegenden Alternativen. Dies gilt vor allem im Bereich von Medieninhalten, da die Grenzen zwischen inhaltlicher Regulierung und staatlicher Zensur fließend und normativ kaum klar zu begründen sind. Zum anderen sind politische Akteure gerade im Zuge zunehmender Medialisierung (Strömbäck 2008) daran interessiert, ihren Zugang zur Agenda und den Kanälen der Massenmedien zu sichern, ohne die gebotene Autonomie von Staat und Medien zu verletzen. Hier bieten sich informelle Regulierungsinstrumente an, die staatlichen und privaten Akteuren die Möglichkeit geben, gemeinsam verbindliche Regeln auch außerhalb der etablierten Institutionen zu setzen. Zudem kann die Inklusion von Stakeholdern und zivilgesellschaftlichen Akteuren die Input-Legitimität medienpolitischer Regulierung erhöhen.

\subsection{Ko-Regulierung, Selbst-Regulierung und Selbst-Organisation}

Wenn nicht staatliche, nicht demokratisch legitimierte Akteure an der Regelsetzung im Medienbereich beteiligt sind, kann dies auf drei verschiedene Weisen geschehen, die sich durch die jeweils beteiligten Akteure unterscheiden. Bei Ko-Regulierung bilden Staat und private/zivilgesellschaftliche Akteure ein Tandem: Staat und Medienakteure finden gemeinsam institutionalisierte Formen gemeinsamer Regelsetzung, Regeldurchsetzung und Sanktionierung bei Regelverstößen. Dadurch kann das Gebot der Pressefreiheit auch bei der Regulierung von Medieninhalten gewahrt bleiben, weil spezifische Vorgaben von Medienakteuren gesetzt und kontrolliert werden. Gleichzeitig stellt die staatliche Beteiligung sicher, dass überhaupt Regulierung stattfindet - während Selbstregulierung weitgehend reaktiv eingesetzt wird, um angedrohter staatlicher Regulierung zuvorzu- 
kommen. In der Praxis bedeutet Ko-Regulierung daher zumeist, dass staatliche Akteure die Implementation von selbstregulierenden Elementen zwingend vorschreiben, kontrollieren und evaluieren (,regulierte Selbstregulierung“). Dies ist z. B. der Fall bei der mexikanischen Wahlberichterstattung, bei der obligatorische Regulierungsinstrumente seit Mitte der 1990er Jahre für parteipolitisch ausgeglichene Medieninhalte sorgen sollen (Klinger 2011a, S. 248-264; Mena 2005). Insgesamt kommt Ko-Regulierung in Italien und Mexiko aber kaum vor, so dass sie hier nicht weiter empirisch analysiert werden kann. Dadurch wird aber in beiden Ländern die Chance vergeben, private Akteure an der Regelsetzung zu beteiligen, ohne staatliche Aufgaben privater Willkür preiszugeben, wie Offe (2009) in Anlehnung an Crouch's Postdemokratie befürchtete.

Selbstregulierung bezeichnet dagegen die Maßnahmen, mit denen private und zivilgesellschaftliche Akteure selbst Regeln für ihre Branche bzw. ihren Tätigkeitsbereich setzen, durchsetzen und sanktionieren. Damit verfolgen sie primär zwei Ziele: zum einen die Abwendung einer möglichen (angedrohten) Intervention des Staates, in dem man die geforderten Regeln selbst einführt und kontrolliert. In manchen Fällen, wie bei den italienischen Journalistenverbänden (ordine dei giornalisti), sollen eigene Regeln auch vor Interventionen des Marktes schützen, indem z. B. das Berufsrecht und das Lohnniveau zentral von den ordine definiert sind. Ein zweiter Grund ist die Qualitätssicherung, auf die ethische Codices und inhaltliche Vorgaben abzielen. Dabei geht es zentral um ,media accountability“, d. h. um alle nicht staatlichen Aktivitäten, die die Verantwortung der Massenmedien gegenüber der Öffentlichkeit definieren und garantieren sollen (Eberwein et al. 2011; Bertrand 2000). Der Nachteil von Selbstregulierung ist, dass sie häufig erst als Reaktion auf staatlichen Druck hin implementiert wird. So sind beispielsweise Presseund Medienräte nicht aus Initiativen aus der Medienbranche selbst heraus entstanden, sondern mit dem Ziel eingesetzt worden, absehbare staatliche Eingriffe in Arbeitsprozesse oder Inhalte zu vermeiden.

Ein Unterbereich von Selbstregulierung ist Selbstorganisation, d. h. die Regel- und Norm(durch)setzung innerhalb von Medienorganisationen und Medienunternehmen. Offe (2009) hat darauf verwiesen, dass Governance nicht vor den Toren von Märkten und Unternehmen haltmachen kann, wenn der Begriff ,,das Gesamt aller nebeneinander bestehenden Formen der kollektiven Regelung gesellschaftlicher Sachverhalte“ (Mayntz 2004: o. S.) beinhalten soll. Hier ist zwar die Reichweite geringer, dafür aber das Regulierungspotenzial größer, weil meist klare Hierarchien, Zuständigkeiten und Pflichtzuschreibungen existieren. Dies schließt an Governance-Konzepte aus der Ökonomie an, wie corporate governance und corporate social responsibility, aber es geht auch um journalistische Qualität und firmeninterne Standards (Hermes 2006).

Durch die Kompetenzen der beteiligten Akteure unterscheidet sich auch die Reichweite der Regelungen, die mittels Ko- und Selbstregulierung implementiert werden können. Während staatliche Medienpolitik und Ko-Regulierung allgemein verbindliche Outputs generieren, ist Selbstregulierung auf die eigene Branche beschränkt (z. B. auf alle Mitglieder eines Journalistenverbandes) und Selbstorganisation gar auf ein einzelnes Medienunternehmen. Wie weiter unten gezeigt wird, bleibt dies nicht folgenlos, wenn es um die Sicherung von Pluralismus in den Medien geht. 


\subsection{Informelle Regulierung; informelle Institutionen in defekten Demokratien}

Informelle Regulierung unterscheidet sich dadurch von Ko- und Selbstregulierung, dass sie in inoffiziellen, formal nicht legitimierten Verfahren entstanden ist; die Regeln nur den beteiligten Akteuren bekannt, nicht-öffentlich, nicht nachlesbar oder prüfbar und nicht einklagbar sind. Ähnlich klingt dies bei Helmke und Levitsky (2012, S. 88-89), die informelle Regeln als ,socially shared rules, usually unwritten, that are created, communicated, and enforced outside of officially sanctioned channels" definieren. Ko- und Selbstregulierung hingegen basieren auf formalen Institutionen und ergänzen staatliche Regulierung (Gesetzgebung).

Dabei ist es wichtig, informelle Institutionen von anderen informellen Verhaltensweisen zu unterscheiden, die allein durch Habitus oder Gewohnheit motiviert sind. Helmke und Levitsky (2012, S. 95-96) gehen auch der Frage nach, aus welchen Gründen informelle Institutionen entstehen und geben drei Antworten: 1) Akteure etablieren informelle Institutionen weil die formellen Institutionen unvollständig oder ineffizient sind; 2) informelle Institutionen dienen als zweitbeste Lösung, wenn formelle Regelsetzung nicht möglich (oder mit höheren Transaktionskosten verbunden) ist; 3 ) informelle Institutionen finden immer dann Anwendung, wenn die mit ihnen verbundenen Ziele und Motivationen in der Öffentlichkeit als inakzeptabel gelten.

Wolfgang Merkel hat für defekte Demokratien auch auf transformationsspezifische Entstehungszusammenhänge verwiesen. So speise sich ein ,illiberaler Code“, der die Entwicklungsdynamik solcher Demokratien beeinflusst, aus Informalisierungstendenzen, die ihre Wurzeln in tradierten politischen Handlungsmustern (,einem vor-demokratischen Erbe des Informalen“, Merkel 2000, S. 3) und der informellen Problembearbeitung in ineffizienten autoritären Regimen haben. Wie später gezeigt wird, ist dies ein entscheidender Faktor in der Medienregulierung in Italien und Mexiko, weil in beiden Ländern informelle Institutionen Tradition haben und spätestens mit der ,partitocrazia/partidocracia“, der Okkupation des Staates durch die Parteien, in politischen Verfahren manifest wurden.

Informelle Regeln stehen nicht zwangsläufig in Konflikt mit formalen Regeln, sondern finden sich oft in Grauzonen und weisen auch auf Reformbedarf hin. Dies gilt insbesondere dann, wenn sie formelle Regulierung ersetzen (substituierende informelle Institutionen, Lauth 2012, S. 56) oder einen kostengünstigen Umweg bieten:

(O)ne explanation for the increasing informalization of modern policy-making is the need to complement - or to compensate for - inefficient formal procedures. In the face of complexity, informal politics can reduce the transaction costs of formal decision-making, and restricting the number of participants in a decision process is one potential short-cut towards agreement. (Reh 2012, S. 74)

Einige Autoren gehen gar so weit, informelle Arrangements nicht nur als einen selbstverständlichen und gewöhnlichen Teil demokratischer Politik zu betrachten, sondern als soziale Interaktionen, die das Hauptgewicht politischen Handelns ausmachen. ${ }^{1}$ Infor-

1 ,The mainstream of scholarly research (...) is almost exclusively aimed at the $10 \%$ of the visible tip of the iceberg of ongoing social processes that is borne by the $90 \%$ invisible ice under the sea`s surface (...)“ (Brie und Stölting 2012, S. 19). 
melle Institutionen müssen also keineswegs schädlich für liberale Demokratien sein, sondern können wichtige Funktionen übernehmen. Ein Problem für die Demokratie entstehe überhaupt erst dann, so Lauth (2012, S. 56), wenn formelle und informelle Regelsysteme in Konflikt miteinander gerieten. Einige informelle Regeln seien tendenziell ,parasitäre' Institutionen, weil sie auf die Existenz formaler Institutionen angewiesen seien, die sie (teilweise) okkupieren und ausbeuten könnten. Auf diesen Aspekt werde ich später zurückkommen, denn er erscheint mir bedeutend für das Verständnis des regulativen Vakuums, das lange in der italienischen und mexikanischen Medienregulierung vorherrschte. Grundsätzlich lässt sich aber festhalten, dass formelle und informelle Institutionen interdependent sind (,like bones and muscles“", Cheng und Womack 1996, S. 327): Informelle Arrangements brauchen auch formelle Ordnung, innerhalb oder außerhalb derer sie bestimmte Funktionen übernehmen, und formelle Regeln können durch informelle Regeln Flexibilität, höhere Effizienz und Problemlösungskapazität gewinnen (Stacey 2010, S. 66-74).

Für den Zweck einer Analyse der informellen Governance-Strukturen im Medienbereich, die weiter unten näher beleuchtet werden sollen, lassen sich zwei Subtypen informeller Regulierung unterscheiden:

1. Regulierungen, die durch vertrauliche inoffizielle Absprachen der beteiligten Akteure entstanden sind (horizontale informelle Regulierung) und

2. Regulierungen, die ein Akteur einem oder mehreren anderen Akteuren in einem informellen Verfahren auferlegt (vertikale informelle Regulierung).

Eine weitere Form ist die Duldung von Akteuren, Strukturen und Handlungen in einem nicht-regulierten Bereich - die aber den Begriff der Regulierung kaum rechtfertigt, weil zwischen den Akteuren keine Beziehungen bestehen, die auf die Herausbildung irgendeiner Ordnung abzielen. Dabei handelt es sich eher um eine zeitlich und räumlich begrenzte Anerkennung einer regelfreien Situation im Sinne einer aktiven Nicht-Regulierung.

\section{Medienpluralismus als Regulierungsziel}

In der Medienpolitik ist Governance vor allem dann ein hilfreiches Konzept, wenn es um Regelsetzung in Bereichen geht, in denen die Handlungskompetenz bzw. die Interventionsmöglichkeiten des Staates begrenzt sind. Dies trifft in besonderem Maße auf Medienpluralismus als Regulierungsziel zu. Pluralistisch können dabei die Medien(eigentums) strukturen (externer Pluralismus) und die Medieninhalte (interner Pluralismus) gestaltet sein. Medienpluralismus bezieht sich demnach sowohl auf die Anzahl der Medienorganisationen in einer Gesellschaft sowie die Vielfalt an verfügbaren medialen Inhalten.

Die Interventionsmöglichkeiten staatlicher Akteure sind in der strukturellen Gestaltung des Medienmarktes deutlich größer als bei den Medieninhalten - so schließt das verfassungsgemäße Gebot der Pressefreiheit nicht nur staatliche Zensur aus, sondern verbietet auch eine staatliche Einmischung, die ein Mehr an Meinungen, Stimmen und Vielfalt in Zeitungen und Rundfunksendungen vorschreiben würde. Mena (2005, S. 28) hat auf den regulativen Zielkonflikt von Pressefreiheit und Medienpluralismus im Kontext 
von Wahlberichterstattung in Großbritannien und Mexiko verwiesen, der zumindest in einem eng negativ ausgelegten, klassisch liberalen Verständnis besteht:

First, media owners have a right to editorial discretion that allows them to exclude from their political comment and reporting any view or position they disagree with. Second, media owners have a right to advocacy, allowing them to proselytize in favor the views they share and against those they disagree with. Third, media owners have full control over their property allowing them to deploy the human and technical resources of their organizations in favor of whichever political cause they deem worth endorsing. (...) (M)edia owners have a right to freely express their political views and to contribute toward the political cause of their choice with the means at their disposal.

Regulative Vorgaben durch staatliche Akteure für Medieninhalte sind demnach heikel auch wenn sie dem Zweck dienen, den Pluralismus an Meinungen zu erhöhen und allen beteiligten Kandidaten oder Parteien Medienpräsenz zu garantieren. Dies ist in einem liberalen Demokratieverständnis nur durch freiwillige Selbstverpflichtungen und Regulierung durch Medienakteure selbst zu erreichen.

Eine Ursache für abnehmenden Medienpluralismus ist die Medienkonzentration - ein ökonomischer Prozess, bei dem sich private kommerzielle Medienunternehmen zu immer größeren Einheiten zusammenschließen. Gründe dafür sind eine mögliche Fixkostendegression beim Zusammenschluss von Anbietern im selben Markt (horizontale Konzentration), die Reduktion von Transaktionskosten durch eine Fusion von vorgelagerten Produzenten und Weiterverwertern (vertikale Konzentration) und diverse andere Vorteile wie Quersubventionierungen, Synergieeffekte und Verbundvorteile durch cross-mediale Konzentration in verschiedenen Marktsegmenten. Konzentrationsprozesse im Medienbereich können zwar bei einer Monopolbildung auch zu Marktversagen führen, sind aber zunächst ökonomisch attraktiv und sinnvoll für Unternehmen, die sich in einem zunehmend krisengeplagten Markt (Blum et al. 2011) bewegen. Aus sozialwissenschaftlicher Perspektive steht man der Medienkonzentration hingegen äußerst kritisch gegenüber: Die Medien seien zu einer ,anti-demokratischen Kraft“ (anti-democratic force) geworden (Mc Chesney 2000), verursachten Schäden in der Politik, Wirtschaft, Kultur und Bildung (Trappel et al. 2002) und keine Demokratie „sollte diese Gefahr riskieren“ (should risk the danger) (Baker 2007, S. XX).

Die Vielfalt von Inhalten und Anbietern im Medienbereich ist zentral für die Entwicklung und die Qualität von Demokratie, was sich nicht nur in der Literatur zur Demokratietheorie niederschlägt (Dahl 1971, Diamond und Morlino 2005, Merkel et al 2003, Merkel und Puhle 1999, Gunther und Mughan 2000 u. a.), sondern auch in etablierten Demokratie-Indices. So gilt den Sustainable Governance Indicators (www.sgi-network. org) Medienpluralismus als eines von drei Kriterien für die Kategorie „Zugang zu Informationen“ in der Messung der Demokratiequalität der OECD- Staaten. Auch bei Freedom House spielt Medienpluralismus eine Rolle - so wird Italien seit 2004 (mit einer kurzen Unterbrechung in den Jahren 2007 und 2008) im Pressefreiheitsindex nur als ,teilweise frei" eingestuft.

Da Medienkonzentration zwar wirtschaftlich sinnvoll, aber politisch nicht erwünscht ist, regulieren die meisten demokratischen Staaten die Medienstrukturen nicht nur über 
Wettbewerbsgesetze, sondern auch durch verbindliche Konzentrationsgrenzen, nach deren Erreichen keine weiteren Fusionen für ein Medienunternehmen mehr möglich sind - mit dem Ziel, marktbeherrschende Stellungen zu vermeiden. Die Festsetzung solcher Konzentrationsgrenzen ist meist willkürlich und problematisch, auch wenig effektiv, weil sie sich an der bestehenden Marktsituation orientieren und oftmals nur den Status quo festschreiben. Ein Beispiel aus der italienischen Medienpolitik illustriert dies: Nachdem das Verfassungsgericht 1994 und 2002 eine Absenkung der Konzentrationsgrenze von 25 auf $20 \%$ des Marktanteils gefordert hatte und dies politisch jahrelang nicht umgesetzt worden war (auch weil Berlusconi und seine Koalitionspartner die nötigen parlamentarischen Mehrheiten verhinderten), löste Berlusconis Regierung diese Vorgabe 2004 mit dem Gasparri-Gesetz so effektiv wie innovativ. Indem die Marktgröße als Berechnungsgrundlage der Konzentrationsgrenzen ausgeweitet wurde (dazu zählten nun neben Presse und Rundfunk auch Kino, Werbung, Lizenzgebühren, das Internet u. a.), konnte die Grenze formell auf $20 \%$ gesenkt werden, während Berlusconis Medienunternehmen weiter wuchsen und die drohende Schließung eines seiner Sender abgewendet war. Der Präsident der italienischen Wettbewerbsbehörde kritisierte in der parlamentarischen Anhörung, dies sei, als ob man bei der Berechnung des Marktanteils von Coca Cola das Leitungswasser mit einbezöge (Klinger 2011a, S. 137). Auch in anderen Fällen und Ländern, in denen partikulare Interessen weniger direkt in die Regulierung von Medienpluralismus hineinspielen, hat sich gezeigt, dass es schwierig ist, strukturelle Konzentrationsgrenzen zu begründen und durchzusetzen (Just 2009; Bornemann 2006). Es handelt sich demnach um ein Politikfeld, in dem nur eine Verschränkung von staatlicher und privater Regulierung, von formellen Institutionen und informeller Regelsetzung zur Herstellung und Sicherung von Medienpluralismus führen kann.

\section{Mexiko und Italien: Fallauswahl}

Der interregionale Vergleich von Italien und Mexiko unterstreicht die Relevanz von Media Governance für die Regulierung von Medienpluralismus. Beide Länder weisen die höchsten Medienkonzentrationsraten innerhalb der OECD und in ihren Regionen auf, beide gelten als defekte Demokratien (Merkel et al. 2003) und enge Verbindungen zwischen politischen und medialen Akteuren sowie ein hoher ,political parallelism“ (Hallin und Mancini 2004, S. 26-33) sind typisch und tief im politischen System und im Mediensystem verwurzelt. So kann auch Mexiko dem polarisiert-pluralistischen Modell zugeordnet werden, das Hallin und Mancini (2004) ursprünglich für die Mediensysteme in Italien und anderen Länder im mediterranen Raum formuliert haben. Informelle Regelsetzung hat in beiden Ländern Tradition und bildet vor den unterschiedlichen Ausprägungen der formalen Herrschaftsstrukturen die Basis für vergleichende Fallstudien.

In Italien und Mexiko dominieren jeweils zwei Medienorganisationen den Rundfunkbereich: die staatliche RAI (Radiotelevisione Italiana) und Berlusconis Mediaset in Italien sowie Televisa und seit 1993 TV Azteca in Mexiko. Beide Akteure kontrollieren zusammen jeweils gut $90 \%$ des Rundfunkmarkts in ihren Ländern - sowohl in Bezug auf die Zuschauerreichweiten als auch der verfügbaren Ressourcen (Klinger 2011a, 
S. 13-34). Über die italienische Rundfunklandschaft heißt es in den Sustainable Governance Indicators 2011:

With regard to television, the system is dominated by a duopoly formed by the stateowned broadcaster and Mediaset, but with other actors (in particular News Corp. and its Sky TV) slowly growing in importance in the cable and satellite sector. (...) Once again, due to the dominance and the importance of television as the nation's and Italians' main information medium, a wide diversity of opinions is not immediately available. Only by consulting electronic media and newspapers, as only a minority do, can media users, consumers and citizens produce for themselves a certain pluralism. (SGI Network 2011)

Auch die Herabstufung auf einen nur „teilweise freien“ Status der Pressefreiheit durch Freedom House 2004 begründete sich durch den mangelnden Pluralismus:

Italy's rating moved downward from Free to Partly Free as a result of high media concentration and increased political pressures on media outlets. The country's free and independent media institutions are threatened by government interference and the highest level of media concentration in Europe. This trend results from the 20-year failure of political administrations to reform the framework for independent journalism and access to information. (Freedom House 2004)

Ganz ähnlich sieht es in Mexiko aus, wo zwei kommerzielle Akteure sich den Rundfunkmarkt teilen und Wettbewerber, auch staatliche Sender, nur eine marginale Rolle spielen. Televisa entstand Mitte der 1960er Jahre und war als kommerzieller Rundfunkmonopolist dem Regime der Partei der institutionalisierten Revolution PRI, die Mexiko von 1929 bis 2000 regierte, durch ein enges informales und kollusives Verhältnis verbunden. Die Regulierung kommerzieller Medien - oder auch die Androhung von staatlicher Regulierung fand nur statt, um inhaltliche Passung mit der Politik der PRI zu garantieren. Die Entstehung eines Monopols wurde dafür billigend in Kauf genommen und staatlich abgesichert. Der informelle Tausch politischer Gefälligkeit gegen weitgehend unbeschränkte Expansion und Marktdominanz sicherte die unangefochtene Hegemonie von PRI im Staat und Televisa im Medienmarkt. 1993 kam TV Azteca hinzu, das im Zuge deregulativer Politik durch Privatisierung von staatlichen Sendern entstanden war - und die Kommerzialisierung (und Depolitisierung) des mexikanischen Rundfunks einläutete.

In beiden Ländern sind es einheimische Medienunternehmen, die den Markt dominieren. Sie sind in sehr hohem Maße und anders, als dies ausländischen Medienkonzernen möglich wäre, in informellen Netzwerken etabliert und verfügen über tradierte enge Beziehungen zu den politischen Akteuren - die ihnen jahrzehntelang dabei geholfen haben, externe Konkurrenten aus dem Markt zu halten. Das ist ein ganz wesentlicher Unterschied zu anderen Fällen, wie z. B. den postkommunistischen osteuropäischen Ländern, in denen hohe Medienkonzentration herrscht, weil westeuropäische, vor allem deutsche und Schweizer Medienkonzerne seit der Transformation dominieren.

In keiner anderen modernen westlichen Demokratie findet sich eine höhere Marktkonzentration und weniger externer Medienpluralismus als in Italien und Mexiko. Gleichzeitig unterscheiden sich die politischen Systeme stark voneinander - wenngleich es sich in beiden Fällen um defekte Demokratien handelt. Mexiko konnte seit der Transition im 


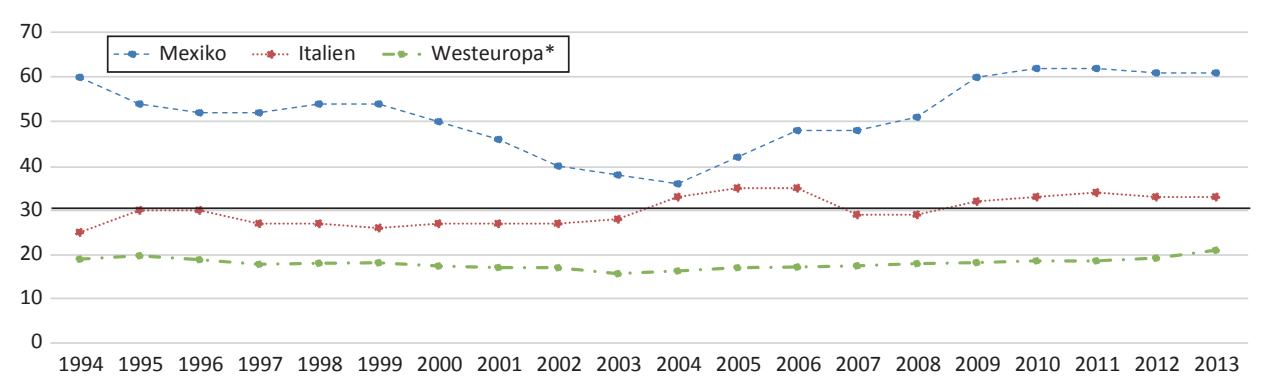

Abb. 2: Entwicklung der Pressefreiheit in Italien und Mexiko 1994-2013. (Eigene Darstellung. Datenquelle: Freedom House Freedom of the Press (Scores and Status 1980-2013, http://www.freedomhouse.org/report-types/freedom-press) Scores: 0-30 free media, 31-60 partly free media, 61-100 not free media. *Western Europe: Andorra, Austria, Belgium, Cyprus (Greek), Cyprus (Turkish), Denmark, Finland, France, Germany, Greece, Iceland, Ireland, Italy, Liechtenstein, Luxembourg, Malta, Monaco, Netherlands, Norway, Portugal, San Marino, Spain, Sweden, Switzerland, Turkey, United Kingdom)

Jahr 2000 nicht alle Defekte abbauen, da vor allem Gewaltakteure den Staat massiv herausfordern. Italien ist hingegen durch verschiedene Fehlleistungen zur defekten Demokratie geworden: In den Sustainable Governance Indicators rangiert Italien 2011 auf Rang 26 (6.3), Mexiko auf Rang 29 (5.5) von 31. In der Kategorie ,access to information“ hat Mexiko (Rang 23) Italien (Rang 29) unterdessen überrundet (Abb. 2).

Wie die Daten des Pressefreiheitsindex von Freedom House zeigen, ist der Rückstand Italiens im Vergleich zu Westeuropa nicht nur temporär, sondern als dauerhaft anzusehen. Mindestens seit 1994 (seit Freedom House Scores vorlegt) bewegt sich die Pressefreiheit in Italien an und jenseits der Grenze zu einem Mediensystem, das nur noch als ,teilweise frei“" eingestuft wird und ca. 10 Punkte über dem westeuropäischen Schnitt liegt. Ein Hauptgrund für diese Situation ist die extreme Medienkonzentration in Italien, wie auch die unzureichende Regulierung insbesondere des Rundfunksektors. Im Vergleich zu einer eher stabilen Defektlage in Italien, zeigt sich die Pressefreiheits-Performance in Mexiko deutlich transformativer. Obschon durchgängig als ,teilweise frei“ eingestuft, verbesserte sich die Pressefreiheit Ende der 1990er Jahre bis 2004 deutlich, um anschließend wieder auf das Ausgangsniveau von 1994 zurückzufallen; in den letzten Jahren wurde sogar die „nicht freie“ Zone erreicht. Vor diesem Hintergrund wird deutlich, dass Italien und Mexiko die „Defektzone“ aus verschiedenen Richtungen betreten haben und beide, wie es scheint, noch länger im Bereich eines nur teilweise freien Mediensystems verharren werden, wobei in Mexiko deutlich gravierendere Probleme vorliegen.

Darüber hinaus zeichnen sich die Mediensysteme in Italien und Mexiko, wie bereits erwähnt, gleichermaßen durch eine unterdurchschnittliche Regulierung der Medien aus. „The narrowness of control of the Italian media ist striking“ postulierte die European Federation of Journalists bereits vor zehn Jahren (EFJ 2003, S. 21), ohne dass sich daran zwischenzeitlich etwas geändert hätte. Sie spielt damit auf das „regulative Vakuum“ im Medienbereich an, einen Mangel an Medienaufsicht, der auch durch zahlreiche Reformen seit 1990 nicht beseitigt wurde. Dies trifft auch in Mexiko zu, wobei hier ein veraltetes Fernseh- und Radiogesetz von 1960 ganze Akteursgruppen unberücksichtigt lässt und 
zudem durch eine deregulative Reform 2006 weiter ausgehöhlt wurde - so dass Tranquinas (1995) Diktum der „savage deregulation“ noch immer gilt.

Die empirischen Daten zu Media Governance in beiden Ländern, die im Folgenden präsentiert und diskutiert werden, entstammen einer Sekundäranalyse bestehender Studien sowie qualitativer Dokumentenanalysen (Codes of Ethics, Webseiten der Journalistenverbände, Dokumente der Selbstdarstellung/Mission Statements) und Experteninterviews. Ausgewertet wurden auch die stenografischen Protokolle von Parlamentsdebatten, die sich um Rundfunkreformen drehten. Letztere werden hier aber nur am Rande berücksichtigt, weil der Fokus dieses Beitrags auf Selbstregulierung und informellen Verfahren liegt. Die Fallstudien sind akteurszentrierte Analysen der Regulierungsprozesse, die sich mit Pluralismus im Rundfunkbereich befassen.

Die Studie bezieht sich nur auf den Rundfunksektor und dort in erster Linie auf das Fernsehen. Dies hat drei Gründe: erstens ist im Fernsehmarkt die Medienkonzentration am höchsten und damit der Regulierungsdruck am stärksten, weil zweitens das Fernsehen in beiden Ländern die (politische) Hauptinformationsquelle der Bürger ist, die vergleichsweise selten Zeitung lesen; und drittens ist die Presselandschaft grundsätzlich und regional übergreifend weitgehend unreguliert, schon weil die Zahl der Presseprodukte im Gegensatz zum begrenzten Rundfunkspektrum nach oben offen ist. Weil nun prinzipiell jeder Bürger und jede Bürgerin eine Zeitung herausgeben, aber nicht auf einer beliebigen Rundfunkfrequenz an ein Massenpublikum senden kann (jenseits von Teil- und Nischenöffentlichkeiten, die im Internet entstanden sind), findet sich Media Governance vor allem im Rundfunkbereich.

Eine weitere Grenze der Studie ist ihr Fokus auf die nationalstaatliche Ebene. Die Verhandlung von Medienpluralismus in lokal oder regional begrenzten Räumen findet keine Berücksichtigung, was sich auch aus der schwach föderalistischen Struktur in beiden Ländern und der Dominanz nationaler Sender begründet.

\section{Media Governance in Italien}

Wie bereits dargestellt, regeln gesetzliche Vorschriften den Rundfunksektor in Italien nur unzureichend. Aber auch Ko- und Selbstregulierung greifen in Italien nur bedingt, wenn es um die Sicherung von Pluralismus geht. In Italien sind ethische Standards im Medienbereich, die auf eine gesellschaftliche Funktion der Medien, Verantwortung und Pluralismus verweisen, nur schwach institutionalisiert. Auch die Professionalisierung und Autonomie der Journalisten ist gering (Hallin und Mancini 2004) - wenngleich die journalistischen Berufsverbände (ordine dei giornalisti) hochgradig formalisierte Zugangskontrollen vornehmen, die enorme Konsequenzen für Journalisten haben. Die Ordine wurden auf staatliche Initiative per Gesetz Nr. 69 am 3. Februar 1963 gegründet, wodurch der Journalismus als Beruf eine staatliche Anerkennung als Profession mit sozialer Relevanz erfuhr. Die Hauptaktivitäten der Ordine liegen in der Kontrolle des Berufszugangs und der Interessenwahrung, zum Beispiel durch Festlegung von Tarifen für journalistische Leistungen. 
Italian journalists have always considered themselves as part of a very special caste. To this day, the profession protects itself by rigorously enforced entrance barriers (...). Being a member of the , ordine dei giornalisti' comes with a number of privileges. Fully employed journalists are not heard of as being fired, not even in cases where serious professional mistakes have been made. (...) During the 1980s quality papers like La Repubblica started to buy heavily into ailing regional dailies. Chain newspapers with regional or local inserts developed with almost no reduction in editorial staff. (OSZE 2003, S. 151)

Die Setzung, Durchsetzung und Sanktionierung von ethischen Standards spielt hingegen keine vergleichbare Rolle (Hallin und Mancini 2004, S. 112). Einen verbindlichen Ethikcode kennen die Ordine nicht. Auf nationaler Ebene gelten elf „deontologische“ Vereinbarungen, die sich zum Teil überschneiden und ergänzen. In diesem Bereich ist Italien ein Nachzügler unter den westlichen Demokratien. Es bestehen keine effektiven Sanktionsmechanismen, die die Einhaltung der normativen Regeln überwachen und Verstöße veröffentlichen. Die Mitgliedschaft in den Ordine ist so zwar an hohe Zugangsbarrieren, aber nicht an die Einhaltungen der normativen professionellen Standards gebunden. Eine effektive Förderung von inhaltlichem Pluralismus - auch wenn dies in einigen Codices angesprochen wird - ist durch diese Maßnahme nicht zu erwarten. Wie Mancini (2000, S. 123) schreibt, kannten einer Studie von 1997 zufolge weniger als ein Drittel der italienischen Journalisten die wichtigsten Ethikcodes und viele lehnten sie ab. Einen Presseoder Medienrat auf nationaler Ebene, der ethische Standards setzt und als Adressat für Beschwerden von Bürgern dient - ähnlich dem Deutschen Presserat oder dem Britischen Press Council - gibt es in Italien nicht.

Journalistische Praktiken und Medieninhalte werden nicht nur von Pressegesetzen und Branchennormen beeinflusst, sondern auch von den Regeln, die Medienunternehmen innerhalb ihrer Organisationen setzen. Im Zeichen eines unternehmensinternen Qualitätsmanagements (Hermes 2006) können so effektive Maßnahmen zur Sicherung eines internen Pluralismus implementiert werden. Die Reichweite dieser Regulierung ist zwar begrenzt, aber da es sich durch die bereits etablierte Medienkonzentration bei RAI und Mediaset um dominante Rundfunkunternehmen handelt, decken sie sowohl den Fernsehals auch Radiojournalismus nahezu komplett ab.

Beide Unternehmen schreiben ihren journalistischen Mitarbeitern Ethikcodes vor. Da Maßnahmen zur Selbstorganisation meist primär den Aspekt der Qualitätssicherung betonen, scheint es bemerkenswert, dass darin jedoch nur sehr wenige Artikel enthalten sind, die die Mitarbeiter auf journalistische Qualität einschwören (Klinger 2011a, S. 223). Dabei orientiert sich die RAI signifikant stärker an einer gesellschaftlichen Verantwortungswahrnehmung und sieht sich auch als Unternehmen in Staatsbesitz als Dienstleister in der Pflicht der Öffentlichkeit. Mediaset fällt im Vergleich dazu deutlich ab, da Gesellschaft, Öffentlichkeit oder Pluralismus keine Rolle bei der internen Regelsetzung spielen und kaum je Erwähnung finden. Hier wird die Chance vergeben, in einem Bereich, in den der Staat kaum formal regulieren kann, inhaltlichen Pluralismus als journalistische Norm zu etablieren. Die möglichen Sanktionen, insbesondere die Disziplinarstrafen, bleiben unspezifisch und diffus. 


\subsection{Informelle Institutionen: Das „Lottizzazione“-System}

Lange bevor die Governance-Debatte begann, haben sich in beiden Ländern informelle Institutionen etabliert, wie das „Lottizzazione“-System in Italien. Ohne jegliche formale Basis werden die öffentlichen Rundfunksender demnach auf Regierungs- und Oppositionsparteien ,verteilt“. Diese bestimmen nicht nur die inhaltliche Ausrichtung, sondern besetzen auch Verwaltungs- und journalistisches Personal.

Die Wurzeln des Lottizzazione („Parzellierung“)-Systems reichen zurück bis in die 1950er Jahre, als sich ein informelles power sharing von Democrazia Cristiana (DC) und Partito Socialista Italiano (PSI) herausbildete, um den starken kommunistischen PCI zu isolieren. Ebenso wie die DC die Nachkriegspolitik in Italien dominierte, war auch der Rundfunksektor von ihrem Parteimonopol geprägt. Obwohl nach dem Zweiten Weltkrieg Pläne bestanden hatten, den staatlichen Rundfunk in Erwartung wechselnder Regierungskoalitionen autonom von Regierung und Parteien zu gestalten, wurde die Staatsferne nie umgesetzt - auch weil sich parallel die Dauerregierung des DC etablierte. ${ }^{2}$ Stattdessen wurde die RAI zum anti-kommunistischen und katholischen Sprachrohr der Regierungskoalitionen, die den organisatorischen Aufbau der RAI kontrollierten und alle Funktionen besetzten, wie etwa den Verwaltungsrat.

Dies änderte sich 1975, als eine Rundfunkreform die Kontrolle der RAI-Sender in die Hände einer Parlamentskommission legte, die von beiden Kammern gewählt wurde und entsprechend die parteipolitische Zusammensetzung der italienischen Legislative spiegelte. Grund dafür war nicht nur eine immer stärkere Opposition und die gesellschaftspolitischen Veränderungen der 1960er Jahre, sondern auch zwei Grundsatzentscheidungen des italienischen Verfassungsgerichtes, das 1974 und 1976 maßgeblich Rundfunkreformen einleitete. Der öffentliche Rundfunk wurde nach wie vor in informellen Verfahren auf zwei RAI-Sender verteilt und die beiden dominierenden Parteien, DC und PSI, übernahmen praktisch die Kontrolle über Strukturen und Inhalte jeweils eines dieser Sender. Mit der Gründung von RAI Tre, der seit 1987 vom PCI administriert wurde, war die Lottizzazione perfekt: der konservative DC kontrollierte RAI uno, die Sozialisten RAI due und die kommunistische Partei RAI tre.

Während die meisten Autoren in der Lottizzazione einen Ausdruck der demokratieschädlichen Verflechtungen von Politik und Medien, von Patronage und Klientelismus sehen (z. B. Padovani 2007), bietet Paolo Mancini (2009) eine weitere Lesart an. Demnach befördere diese informelle Übereinkunft der Parteien einen zumindest politischen Medienpluralismus und garantierte gleichzeitig im Zeichen der sich dynamisierenden Medialisierung, dass alle wesentlichen politischen Parteien und ihre gesellschaftlichen Partner (z. B. Gewerkschaften) gesicherten Zugang zur Agenda der staatlichen Medien haben.

(I)t is a way of assuring the different political groups that the circulation of their opinions and point of view (pluralism) is certain. (...) In this perspective, lottizzazione can be seen as a recognition of the existence of many groups (exclusively political

2 Für ausführliche und detaillierte Darstellungen zur italienischen Mediengeschichte und Rundfunkentwicklung (Vgl. Hibberd 2008; Mancini 2000; Monteleone 2006; Padovani 2007). 
groups in Italy's case) in competition and their need to express and circulate their points of view. Public service broadcasting offers such groups the opportunity of spreading their opinions, giving shape to an ideal public media sphere. (Mancini 2007, S. 113 und 115)

Entgegen der Intentionen der Reformakteure legte dieses Modell aber nicht den Grundstein für autonomeren und pluralistischeren öffentlichen Rundfunk, sondern für stärkeren parteipolitischen bias und eine organisatorische Aufblähung der RAI:

The whole organizational structure of RAI became more geared to a political logic rather than providing a public service. With the system of lottizzazione, Italy's premier media institution lost any ideas of political autonomy and impartiality it may have haboured before the reform process began. (...) One effect of having three near-identical networks was that the television-making process was in part triplicated, and this therefore created unnecessary additional costs. (Hibberd 2008, S. 76)

Obwohl das italienische Rundfunksystem bis heute, mindestens aber bis zur Neuordnung des Parteiensystems in den 1990er Jahren, nach diesen parteipolitischen Verteilungsregeln funktioniert, bleiben sie rein informal und sind nirgendwo niedergeschrieben. Die Gründung von RAI Tre und ihre Übereignung in die Verantwortung des PCI 1987 wurde gar bei einem damals geheimen Abendessen in einem römischen Restaurant beschlossen, an dem Biagio Agnes, der Generaldirektor der RAI (DC), Enrico Manca, der Vorsitzende des RAI Verwaltungsrats (PSI) und Walter Veltroni als Medienbeauftragter des PCI teilnahmen - die dieses Treffen und seine Bedeutung später nicht dementierten (Mancini 2009).

Die Lottizzazione ist eine Institution der horizontalen informellen Regulierung, bei der im Zeichen der partitocrazia die politischen Parteien den Staat beherrschen und den staatlichen Rundfunk unter sich aufteilen. Der kommerzielle italienische Fernsehmarkt entwickelte sich ebenfalls in einer rechtlichen Grauzone, weil die Politik es nach der prinzipiellen Zulassung von Privatsendern durch das Verfassungsgericht über eine Dekade lang versäumte, formal Regeln zu setzen. Berlusconis Privatsender liefen seit Beginn der 1980er Jahre, wurden aber erst mit dem Mammí-Gesetz von 1990 legalisiert. In den Nischen abseits formeller Regulierung setzen sich die ersten regionalen Sender fest. Berlusconis heutige Dominanz im Fernsehmarkt basiert auf dieser informellen (Nicht-) Regulierung, weil er sich als erfolgreicher Bauunternehmer das ökonomische Risiko leisten konnte, in einer solchen regulativ unsicheren Situation zu investieren und bestehende regionale Konkurrenzsender aufzukaufen.

Lottizzazione spiegelt sich auch in der Mediennutzung wieder. Wenn sich Parteien im Zuge der Medialisierung die Kontrolle über Radio- und Fernsehsender sicherten, waren entsprechend auch die Nachrichtenprogramme parteipolitisch gefärbt und ermöglichten dem Publikum eine selektive Medienzuwendung. In diese tradierte Verhaltensweise spielt nun auch Mediaset hinein, deren Sender klar die politische Position von Berlusconis Koalitionen abbilden. Daten aus dem Wahlkampf 2006 belegen, dass sich Wähler der linken Unione ganz überwiegend den Telegiornali der RAI zuwenden (82\%, davon $35 \%$ dem linken, vormals PCI-assoziierten RAI tre), während Wähler des rechten Bündnisses 
Casa della Libertà zu 57\% lieber die Nachrichten bei Mediaset schauten (Cristadoro 2007).

Während die informelle Praxis der Lottizzazione im Zeitverlauf zumindest für eine mediale Abdeckung der dominanten parteipolitischen Strömungen sorgte, scheitern Selbstregulierung und Selbstorganisation am mangelnden Regulierungswillen der privaten Akteure. Auch wenn Selbstregulierung eine von der Politik zugewiesene Aufgabe der Branchenvertreter in den Ordine ist und Medienunternehmen intern große Gestaltungsmöglichkeiten haben, spielen Pluralismus und gesellschaftliche Aufgaben der Medien nur eine marginale Rolle in der italienischen Media Governance.

\section{Media Governance in Mexiko}

Mexikos Mediensektor ist nicht nur in Sachen Pluralismus unterreguliert. Vielmehr ist es den politischen Akteuren seit der Transition, der Abwahl des PRI im Jahre 2000, nicht gelungen, die Medienregulierung an die Bedürfnisse und Erfordernisse eines demokratischen Gemeinwesens anzupassen. Das Radio- und Fernsehgesetz von 1960 wurde erst 2006 in der berüchtigten „Ley Televisa“ reformiert, in der weiter dereguliert wurde und das regulative Vakuum erhalten blieb, von dem staatliche und zivilgesellschaftliche Medien betroffen sind.

Ähnlich wie in Italien weist der Journalismus als Beruf in Mexiko nur einen geringen Grad an Autonomie und Professionalisierung auf. Zudem handelt es sich um ein hochgefährliches Berufsfeld: Seit 2003 wurden 80 Journalisten ermordet, 17 von ihnen sind „verschwunden“ (Reporter ohne Grenzen 2013). Damit nimmt Mexiko, zusammen mit dem Irak und nach Pakistan, den zweiten Rang der für Journalisten gefährlichsten Länder der Welt ein. Diese Zahlen weisen darauf hin, dass mexikanische Journalisten in einem weitgehend unregulierten Bereich agieren, in dem noch nicht einmal ihre körperliche Unversehrtheit hinreichend geschützt ist. Der Zugang zur Profession ist nicht formalisiert. Journalist ist, so steht es im Ethikcode des Dachverbandes der mexikanischen Journalistenverbände FAPERMEX (Federación de Asociaciones de Periodistas Mexicanos), wer dem Journalismus in jedweder Form beruflich in regulärer Beschäftigung oder als Haupterwerbsquelle nachgeht. Zwar nehmen mexikanische Journalisten sich selbst nicht in erster Linie als Arbeitnehmer, sondern als Mitglieder einer Profession mit besonderem Status - als „Vierte Gewalt“ - wahr, dennoch führt, so argumentiert Fuentes Muñiz (2008, S. 29) gerade diese Einstellung zu einer Statusgruppe von Individualisten, die zum größten Teil nicht nur wenig Interesse für professionelle Organisationen oder Gewerkschaften zeigten, sondern diese in ihrer Mehrheit ablehnten.

Eine Selbstregulierung der Medien findet in Mexiko allenfalls auf dem Papier statt, es existieren jedoch keine Institutionen, die ethische Standards durchsetzen oder sanktionieren und die Interessen der Profession nach außen vertreten. Die interne Struktur der Medienbranche weist einen losen Netzwerkcharakter auf; Medienunternehmer und Journalisten agieren jeweils in eigenen Verbänden. Die Liste an Journalistenverbänden ist lang. Der Dachverband FAPERMEX führt 99 regionale Verbände, davon 16 allein in der Hauptstadt. Hinzu kommt das 1995 gegründete Nationale Journalistennetzwerk (Red Nacional de Periodistas) mit 1500 Mitgliedern - das aber nicht einmal über eine eigene 
Webseite verfügt; auch darin spiegelt sich der schwache Grad an Institutionalisierung der Medienbranche in Mexiko.

Insgesamt zeigen sich Mexikos Journalisten und Medieneigentümer sehr vernetzungsfreudig - wenngleich diese Aktivitäten bisher zu keiner effektiven Institutionalisierung von Selbstregulierungsmechanismen führten. Auch der Verband der kommerziellen Medienunternehmer Cámara Nacional de la Industria de Radio y Televisión (CIRT) installierte bisher keinen eigenen Ethikcode, dafür aber eine Ethikrichtlinie als Grundlage für Ethikcodes innerhalb der Mitgliedsunternehmen und strukturiert damit die Selbstorganisation der Medienunternehmen vor. Die Ethik-Richtlinie des CIRT war, dies illustriert den bereits beschriebenen reaktiven Charakter von Selbstregulierung, bei einem Symposium der Mitglieder 1998 beschlossen worden, auf dem sich die mexikanische private Rundfunkindustrie gegen eine Reform positionierte, die das Recht auf öffentliche Information (derecho de la informacion) implementierte (Martínez 2009, S. 29). Einen Presse- oder Medienrat, der verbindliche Normen vorgibt, ihre Einhaltung überwacht und Verstöße sanktioniert oder ein Ombudsverfahren für Journalisten gibt es auch in Mexiko nicht.

Ein Vergleich der Ethikcodes von Televisa und TV Azteca verdeutlicht die großen Unterschiede, die in der Selbstorganisation von Unternehmen innerhalb der gleichen Branche bestehen können. Dabei ist bereits die Existenz von Ethikcodes in Medienorganisationen in Mexiko ein Fortschritt, da im Land zwar 730 Fernsehkanäle, 1488 Radiosender und 340 Tageszeitungen existieren, aber nur 30 interne Ethikcodes (Martinez 2009). Während in beiden Fällen viel Wert auf die Verantwortung der Mitarbeiter gegenüber dem Unternehmen gelegt wird, setzt zumindest TV Azteca auch Regeln mit Bezug auf journalistische Qualität und gesellschaftliche Verantwortung (allein der wahrheitsgemäßen und objektiven Berichterstattung widmen sich sechs Unterartikel, Klinger 2011a, S. 223).

Der Ethikcode von TV Azteca zeigt, wie stark Medienunternehmen sich in die inhaltliche Ausgestaltung des Programms einbringen können - auf eine Weise, die staatlichen Akteuren in Demokratien gänzlich unmöglich wäre. In einem Glossar, dass dem Ethikcode beigefügt ist, sind 39 Themen und ihre ,adäquate“ Behandlung aufgelistet, darunter Abtreibung, Scheidung, Staat, Regierung, Tod, Freiheit, Religion, Vergewaltigung usw. Auch enthält der Ethikcode von TV Azteca das Recht auf Gegendarstellung (derecho de replica), das erst seit der Verfassungsreform 2007 verbindlich per Gesetz eingefordert wird. Dies verdeutlicht, dass Selbstorganisation und Selbstregulierung einfacher und flexibler Regeln setzen können und so auch Regeln einführen können, für die es im politischen Prozess (noch) keine Mehrheiten gibt. In der Praxis ist ein solches proaktives Vorgehen jedoch selten. Hier bestünde also die Möglichkeit, verbindliche Regeln für einen inhaltlichen Pluralismus in der Berichterstattung zu setzen - die aber versäumt wird.

\subsection{Informelle Regulierung - Radios comunitarias}

Bereits erwähnt wurde, dass zivilgesellschaftliche Medien ein regulatives Vakuum erfahren - die Abwesenheit von formellen Institutionen und Regeln, die ihre Existenz anerkennen und strukturieren. Dies ist ein Fall von vertikaler informeller Regulierung, bei dem staatliche Akteure durch Nichtregulierung solche Medienakteure einer Situa- 
tion aussetzen, in denen sie weder die Regeln kennen, die zu ihrer formalen Anerkennung, einer Sendelizenz oder staatlichen Ressourcen führen, noch überhaupt über legalen Akteursstatus verfügen. Die mexikanische Mediengesetzgebung unterscheidet staatliche und private kommerzielle Rundfunksender - zivilgesellschaftlich getragene Sender, freie Radios, Bürgersender, Sender, die an Universitäten, Stiftungen oder NGOs angebunden sind, operieren folglich in einem informellen Rahmen, einer legalen Grauzone.

Zivilgesellschaftlich getragene Bürgerradios (radios comunitarias) gibt es in Mexiko seit über 40 Jahren (ausführlich zur Geschichte: Calleja und Solis 2005). Es handelt sich dabei zumeist um kleine lokale Sender, die von engagierten Laien und Aktivisten in ihrer jeweiligen Nachbarschaft, ihrer Stadt oder Region ohne kommerzielles Interesse betrieben werden. Es greift dabei zu kurz, sich diese Radios als ethnisch, indigen, ländlich, arm und marginalisiert vorzustellen, vielmehr gibt es auch Bürgerradios in großen Städten, die sich an junge Akademiker richten, über das Internet senden und ein internationales Publikum ansprechen. Die genaue Zahl der Bürgerradios in Mexiko ist nicht bekannt, Schätzungen der mexikanischen Vertretung des Weltverbandes der Bürgerradios (AMARC) gehen in die Tausende (Klinger 2011b). Legal und formalrechtlich betrachtet existieren diese Sender überhaupt nicht, weil das Fernseh- und Radiogesetz von 1960 nur kommerzielle und staatliche Sender kennt und auch die deregulative Reform von 2006 diese Lücke nicht füllte.

Dies verweist auf Merkels, ,illiberalen Code“ - informelle Institutionen und Praktiken, die als Erblast des autokratischen Regimes die unkonsolidierte Demokratie belasten und zu demokratischen Defekten führen. Die „Erblast“ sind dabei keineswegs die Bürgerradios, sondern der Zustand ihrer absichtsvollen Negierung durch staatliche Institutionen, die den Bürgerradios einen legalen Akteursstatus verweigern - zugunsten einer informellen und willkürlichen Regulierung. Waren Bürgerradios unter der autoritären PRIHerrschaft toleriert (oder: ignoriert) worden, kündigte die konservative Fox-Regierung ab 2002 diese informelle Duldung und begann, Bürgerradios zu kriminalisieren und zu schließen. Bürgerradios galten fortan als klandestine Piraten- oder Guerillasender:

Wir kämpfen gegen Sender, die weder eine Konzession [für kommerzielle Sender, UK] noch eine Lizenz [für staatliche Sender, UK] haben, und wir werden nicht aufhören, bevor jeder operierende Sender entweder eine Konzession oder Lizenz hat - oder eben nicht sendet. Jorge Alvares Huth, Vize-Minister für Kommunikation und Transport ${ }^{3}$

Es tut mir leid, aber wir können weder mit Verbrechern sprechen noch sie verteidigen; als Behörde haben wir die Pflicht, Vergehen zu verfolgen. Eine Lösung des Themas der Bürgerradios zu suchen wäre, als ob einer jemanden umbringt und dann die Behörden darum bittet, nichts zu unternehmen. (Beamter des Ministeriums für Kommunikation und Transport SCT, 2000) ${ }^{4}$

3 "Estamos combatiendo a las estaciones que no tienen concesión o permiso y no vamos a parar hasta que toda estación que opere tenga una concesión o permiso, y si no, que no opere.” (El Universal, 09.10.2003, S. B7).

4 "Lo siento pero nosotros no podemos hablar ni defender a delincuentes, como autoridad tenemos la obligación de perseguir los ilícitos, pedir una solución al tema de las radios comunitarias 
Zwischen 2004 und 2010 erhielten 19 Stationen eine Sendelizenz. Dies war jedoch nur ein Ausnahmeerfolg der Bürgerradios und ihrer Interessenvertreterin AMARC in ihrem politischen Einsatz für eine rechtliche Anerkennung. Es wurden einzelne Sender lizensiert, ohne dass verbindliche, allgemein gültige formelle Regeln implementiert wurden, nach denen auch andere Sender solche Lizenzen beantragen können. Die Antragstellung blieb willkürlich und intransparent. So verlangten die Behörden in manchen Fällen hohe finanzielle Sicherheitsgarantien, mit denen die Bürgerradios gewährleisten sollten, keine Werbung zu senden oder Sponsorengelder anzunehmen (Calleja und Solís 2005, S. 82) - ohne jedwede regulative Grundlage für diese Forderungen. Auch der Antragsweg für Sendelizenzen ist noch immer intransparent.

Bürgerradios bleiben bislang informell regulierte Medienorganisationen. Dies hat dramatische Konsequenzen für diese Sender, da sie so keine Rechtssicherheit bei der Akquise von Ressourcen haben und mit der Abschaltung des analogen Sendespektrums auch keinen Weg in die digitalen Sendepakete finden können. Die Situation der mexikanischen Bürgerradios illustriert die weiter oben bereits angesprochene Frage der Demokratieverträglichkeit informeller Regulierung: Während die Duldung und das informelle Zugeständnis einzelner Sendelizenzen auf den substituierenden Charakter informeller Regulierung verweisen, wird gleichzeitig auch deutlich, dass der flexible und kostengünstige Einsatz solcher Regelungen hier der Implementierung einer dauerhaften formalrechtlichen Lösung im Wege steht. Während die zuständigen Behörden durch einzelne Lizenzvergaben versuchen, den politischen Druck zu verringern, mit dem die Bürgerradios und ihre Interessenvertreter auf eine Reform der Mediengesetzgebung drängen (unter Verweisen auf Menschenrechte und internationale Regelungen), gelingt es den politischen Akteuren bisher nicht, eine inklusivere Medienregulierung zu etablieren. Zwar hat sich die Position der Regierung den Argumenten der radios comunitarias geöffnet (Klinger 2011b), dennoch versuchen kommerzielle Anbieter weiterhin, eine inklusivere formale Ordnung des Mediensektors zu verhindern, unter der sie mit den zivilgesellschaftlichen Akteuren um Ressourcen konkurrieren müssten.

\section{Diskussion}

Media Governance ist als wissenschaftliches Konzept ein vielversprechender Ansatz zum Verständnis der oft informellen Praktiken zwischen staatlichen und privaten Akteuren, die in der klassischen Policy-Forschung häufig übersehen werden. Selbstregulierung, Selbstorganisation und informelle Regeln und Praktiken führen jedoch in beiden Ländern absehbar nicht zu einer effektiven Regulierung von Medienpluralismus. Dies liegt zum einen darin begründet, dass die kollusive informelle Regulierung, also das unerlaubte Zusammenarbeiten zulasten Dritter, die Schaffung transparenter Regeln für neue Akteure auf dem Medienmarkt behindert. Zum anderen scheitert die medieninterne Pluralismusförderung dauerhaft am mangelnden Problembewusstsein und geringer Professionalisierung innerhalb der Branche sowie der Wahrnehmung von Medien als vornehmlich ökono-

seria tanto como que alguien cometiera un asesinato y se le pidiera a las autoridades no hacer nada." (Zitiert in Calleja und Soliz 2005, S. 70). 
mische Güter ohne öffentliche Relevanz durch die dominierenden medialen Akteure. Eine Ausnahme dabei ist die RAI, was auf die besondere Rolle staatlicher Medien mit öffentlich-rechtlichen Anlehnungen verweist. Der reaktive Charakter von Ko- und Selbstregulierung schwächt das Potential dieser Mechanismen, wenn staatliche Akteure ihre Funktion als Regulierer nicht annehmen bzw. nicht durchsetzen und so die Funktionalität von Ko - und Selbstregulierung als ,weiche“ Alternative katalysieren. Wenn nicht die glaubwürdige Drohung von staatlichen Akteuren besteht, Regulierungsziele (wie Medienpluralismus) notfalls per Gesetz durchzusetzen, fehlt es den privaten Akteuren an Anreizen, sich selbst Regeln und Beschränkungen aufzuerlegen. Media Governance kann „Media Government“ mithin sinnvoll erweitern, aber nicht ersetzen. Vielmehr müsste ein Konsens hinsichtlich der Regulierungsziele zwischen staatlichen und privaten Akteuren hergestellt werden, bevor Ko- und Selbstregulierung greifen können.

Die starke Präsenz und langfristige Wirksamkeit von informellen Praktiken und Institutionen in der Medienregulierung verdeutlicht aber auch die Bedeutung des Informellen für die Governance-Forschung. Während sich der Fokus der Medienpolitikforschung lange auf Steuerung und Gesetzgebung begrenzt hat und sich erst seit Kurzem auch auf private Akteure „horizontal“" ausweitet, wurde informelle Regulierung bislang praktisch ausgeblendet. Gerade mit Blick auf Italien scheint dies erstaunlich, denn dort bilden informelle Regelungen keineswegs einen marginalen Bereich der Medienregulierung, sondern das Grundprinzip der Ordnung des staatlichen Rundfunks basiert allein auf informellen Praktiken und Institutionen. Auch der private Rundfunk ist bis Anfang der 1990er Jahre nur informell reguliert worden - wodurch diese informellen Regelungen in der Entstehungszeit privaten Fernsehens den Grundstein für ein späteres privates Monopol der Berlusconi-Sender gelegt haben. In Anlehnung an Helmke und Levitsky (2012, S. 95-96) entstanden die informellen Institutionen hier, weil politische Parteien sich den Zugang zum Rundfunk sichern wollten, diesen aber später auch mit anderen Parteien teilen mussten. Daher wurde ein für die Parteien zufriedenstellendes Arrangement gefunden, ohne auf die öffentliche Akzeptanz dieser parteipolitischen Bemächtigung des staatlichen Rundfunks angewiesen zu sein.

Informelle Regulierung ist ebenfalls ein zentrales Thema in defekten Demokratien, die die Erblasten eines autoritären Regimes tragen und durch unzureichende Reformen auch einen Mangel rational-legaler Herrschaft in der Medienregulierung reflektieren. Dies ist der Fall in Mexiko, wo noch zwölf Jahre nach der Transition ein Mediengesetz in Kraft ist, das in den 1960er Jahren erlassen wurde, um das politische Monopol des PRI und das private Medienmonopol Televisas gegenseitig abzusichern. Dies führt dazu, dass ein ganz wesentlicher Teil der Medienakteure, die es in demokratischen Massendemokratien gibt, in einer Grauzone informeller Regulierung agieren muss. Sämtliche zivilgesellschaftliche Medien (die also weder staatlich, noch privatrechtlich-kommerziell strukturiert sind) unterliegen wenn überhaupt informeller Regulierung - weil sie formalrechtlich nicht existieren. So entstehen informelle Institutionen, weil die formalen Institutionen unvollständig und ineffizient sind, und es keine Mehrheiten für eine entsprechende Reform der formalen Regelsetzung gibt. An diesem Fall wird deutlich, dass die formalen Bereiche von Governance nicht alle Bereiche gesellschaftlicher Realität abdecken und sich wissenschaftliche Analysen daher auch nicht auf formale Governance beschränken können. Dies ist vor allem überall dort der Fall, wo informelle Institutionen und Praktiken Tradition 
haben, tief im politischen System verwurzelt sind und politischen Akteuren immer dann ein Ausweichen auf vermeintlich ,softe“ Regelungen ermöglichen, wenn die Einführung oder Reform formaler Regeln schwierig, langwierig und konfliktbehaftet ist. In diesem Sinne wirken informelle Regelungen parasitär, weil sie auf unzureichenden oder überalterten formalen Regelungen basieren und deren Bestehen absichern.

Problematisch bleiben die methodischen Herausforderungen informaler Regulierungspraktiken. Die Entstehung horizontaler informeller Regulierung durch vertrauliche Absprachen der beteiligten Akteure ist nur äusserst selten in belastbarem Datenmaterial hinterlegt. Teilweise ist es möglich, dass beteiligte Akteure mit einem gewissen historischen Abstand als Zeitzeugen oder Experten zur Datengewinnung beitragen oder Archive Briefe und anderes Material bereithalten. In der Regel jedoch wird die Quellenlage dürftig sein, weil die Nicht-Dokumentation ein zentrales Merkmal horizontaler informeller Regulierung ist. Im Falle der italienischen Lottizzazione ist informelle Regulierung relativ gut dokumentiert, weil die Strukturen, Funktionsweisen und Prozesse innerhalb eines staatlichen Rundfunkmonopols beobachtbar sind - allein die formalrechtliche Grundlage, eine Niederschrift jener Spielregeln, die der institutionellen Ordnung zugrunde liegen, fehlt.

Im Falle von vertikaler informeller Regulierung besteht hingegen eine Asymmetrie zwischen den beteiligten Akteuren. In diesen Fällen ist es möglich, dass diejenigen Akteure, die in einem informellen Verfahren Regeln auferlegt bekommen oder gegen informelle Nicht-Regulierung mobilisieren, öffentlich Stellung nehmen, ihre Situation skandalisieren und die Informalität sichtbar werden lassen. Dadurch ist es einfacher, vertikale informelle Regulierung wissenschaftlich zu untersuchen, wenngleich sich die Akteurs-Asymmetrie in einem interessegeleiteten bias der Quellen und Interviewpartner ausdrücken kann (das ist häufig auch der Fall in der Literatur zu den mexikanischen radios comunitarias). Die genannten forschungspraktischen Probleme erschweren eine Analyse informeller Institutionen und Praktiken ganz erheblich. Dass sie dennoch bestehen, ob als Paralleluniversen oder Ergänzungen zu formaler Regulierung, sollte in GovernanceStudien Berücksichtigung finden. Gerade im Bereich der Media Governance bereitet der sehr begrenzten Handlungsrahmen, den politische Akteure vorfinden, einen fruchtbaren Boden für informelle Regulierung.

\section{Literatur}

Baker, C. Edwin. 2002. Media, markets, and democracy. Cambridge: Cambridge University Press. Baker, C. Edwin. 2007. Media concentration and democracy. Why ownership matters. Cambridge: Cambridge University Press.

Benz, Arthur, und Nicolai Dose, Hrsg. 2010. Governance - Regieren in komplexen Systemen. 2. Auf. Wiesbaden: VS- Verlag für Sozialwissenschaften.

Bertrand, Claude Jean. 2000. Media ethics and accountability systems. Brunswick, N.J.: Transaction Publishers.

Blum, Roger, Hein Bonfadelli, Kurt Imhof, und Otfried Jarren, Hrsg. 2011. Krise der Leuchttürme öffentlicher Kommunikation. Vergangenheit und Zukunft der Qualitätsmedien. Wiesbaden: VS- Verlag für Sozialwissenschaften. 
Borneman, Roland. 2006. Wie die KEK gefühlte Meinungsmacht in Eingriffskompetenz umrechnet. MultiMedia und Recht 5: 275-279.

Brie, Michael, und Erhard, Stölting. 2012. Formal institutions and informal institutional arrangements. In International Handbook on Informal Governance, Hrsg. Thomas Christiansen und Christine Neuhold, 19-39. Cheltenham: Edward Elgar.

Calleja, Aleida, und Beatriz Solís. 2005. Con Permiso. La Radio Comunitaria en Mèxiko. Mèxico: DF.

Cheng, Tun-jen, und Brantly Womack.1996. General reflections on informal politics in East Asia. Asian Survey 36 (3): 320-337.

Cristadoro, Aldo. 2007. Mass media e orientamento politico: Analogie e differenze fra gli elletori italiani. In La maratona di Prodi e los sprint di Berlusconi. La campagna elettorale 2006, Hrsg. Paolo Mancini, 181-199. Roma: Carocci.

Dahl, Robert A. 1971 Polyarchy. Partizipation und Opposition. New Haven: Yale University Press.

Diamond, Larry, und Leonardo Morlino. 2005. Assessing the Quality of Democracy. Baltimore: Johns Hopkins University Press.

DiBella Roldán, Julio. 2005. Un paso importante, un primer paso. In Democracia y medios públicos, Hrsg. La Red de Radiodifusoras y Televisoras Educativas y Culturales de México, 229233. A.C. México, D. F.

Donges, Patrick, Hrsg. 2007. Von der Medienpolitik zur Media Governance? Köln: Herbert von Halem Verlag.

Eberwein, Tobias, Susanne Fengler, Epp Lauk, and Tanja Leppik-Bork. 2011: Mapping Media Accountability - in Europe and Beyond. Köln: Herbert von Halem Verlag.

EFJ, European Federation of Journalists. 2003. European Media Ownership: Threats on the Landscape. A survey of who owns what in Europe. Brüssel (September 2002, Update Januar 2003). http://europe.ifj.org/assets/docs/230/189/d1085e6-54424bd.pdf. Zugegriffen: 06. Mai 2014.

Fabbrini, Sergio. 2008. Conflict of interest in Italy. The case of a media tycoon who became prime minister (2001-2006). In Conflict of Interest and Public Life, Hrsg. Christina Trost und Alison Gash, 188-212. Cambridge: Cambridge University Press.

Freedom House. 2004. Freedom of the Press. Italy. http://www.freedomhouse.org/report/freedompress/2004/italy\#.U0rciFe6iFA. Zugegriffen: 13. April 2014.

Fuentes Muñiz, Manuel. 2008. Sin Derechos Laborales. In Condiciones laborales de las y los periodistas en México, un acercamiento, eds. CIMAC/FES, México, D. F. http://library.fes.de/ pdf-files/bueros/mexiko/06447.pdf. Zugegriffen: 19. Juli 2012.

Gunther, Richard, und Anthony Mughan. 2000. Democracy and the Media. Cambridge: Cambridge University Press.

Hallin, Daniel C., und Paolo Mancini. 2004. Comparing media systems. The models of media and politics. Cambridge: Cambridge University Press.

Helmke, Gretchen, und Steven Levitsky. 2012. Informal institutions and comparative politics: a research agenda. In International Handbook on Informal Governance, Hrsg. Thomas Christiansen and Christine Neuhold, 85-113. Cheltenham: Edward Elgar.

Hermes, Sandra. 2006. Qualitätsmanagement in Nachrichtenredaktionen. Köln: Herbert von Halem Verlag.

Hibberd, Matthew. 2008. The media in Italy. Maidenhead: Open University Press.

Jarren, Otfried, und Donges, Patrick. 2007. Massenmedien. In Handbuch Governance. Theoretische Grundlagen und empirische Anwendungsfelder, Hrsg. Arthur Benz, Susanne Lütz, Uwe Schimank, und Georg Simonis, 452-461. Wiesbaden: VS Springer.

Just, Natascha. 2009. Measuring media concentration and diversity: New approaches and instruments in Europe and the USA. Media, Culture \& Society 31 (1): 97-117.

Kiefer, Marie-Luise. 1999. Das Rundfunkpublikum als Bürger und Kunde. In Rundfunkpolitik in Deutschland. Wettbewerb und Öffentlichkeit, Hrsg. Dietrich Schwarzkopf, 701-744. München: dtv. 
Klinger, Ulrike. 2011a. Medienmonopole in defekten Demokratien. Baden-Baden: Nomos.

Klinger, Ulrike. 2011b. Democratizing media policy: Community radios in Mexico and Latin America. Journal of Latin American Communication Research 1(2). www.alaic.net/journal/index. php/jlacr/article/view/4/9. Zugegriffen: 19. Juli 2012.

Lauth, Hans-Joachim. 2012. Informal governance and democratic theory. In International Handbook on Informal Governance, Hrsg. Thomas Christiansen und Christine Neuhold, 40-64. Cheltenham: Edward Elgar.

Mancini, Paolo. 2000. How to combine media commercialization and party affiliation: The Italian experience. Political Communication 17: 319-324.

Mancini, Paolo. 2007. La Lottizzazione of Italian RAI. Between Pluralism, Consociational Democracy and Clientelism. In Media Industry, Journalism Culture and Communication Policies in Europe, Hrsg. Hans Bohrmann et al., 107-125. Köln: Herbert von Halem Verlag.

Mancini, Paolo. 2009. Elogio della Lottizzazione. Rom: Laterza.

Martínez, Raul Omar. 2009. Codigos de ética periodistica en México. México, D. F: Fundación para la Libertad Expresión.

Mayntz, Renate. 2004. Governance Theory als fortentwickelte Steuerungstheorie? MPIfG Working Paper 04/1. http://www.mpifg.de/pu/workpap/wp04-1/wp04-1.html. Zugegriffen: 21. Juni 2012.

Mayntz, Renate. 2008. Von der Steuerungstheorie zur Global Governance. In Governance in einer sich wandelnden Welt, Hrsg. Gunnar Folke Schuppert und Michael Zürn, PVS Sonderheft 41:65-76. Wiesbaden: VS Verlag für Sozialwissenschaften.

McChesney, Robert W. 2000. Rich Media, Poor Democracy. Communication politics in dubious times. New York.

Mena, David. 2005. When practice oversteps the limits of theory: An assessment of fair media coverage for political parties in Great Britain and Mexico. In Review of Policy Research 22 (1): $27-48$.

Merkel, Wolfgang, und Croissant, Aurel. 2000. Formale und informale Institutionen in defekten Demokratien. Politische Vierteljahresschrift 41 (1): 3-30.

Merkel, Wolfgang, und Puhle, Hans-Jürgen. 1999. Von der Diktatur zur Demokratie. Opladen: Westdeutscher Verlag.

Merkel, Wolfgang, Hans-Jürgen Puhle, Aurel Croissant, Claudia Eicher, und Peter Thiery, Hrsg. 2003. Defekte Demokratien. Theorien und Probleme. Opladen: Leske + Budrich Verlag.

Monteleone, Franco. 2006. Storia della Radio e della Televisione in Italia. Costume, Società e Politica. Venedig: Marsilio.

Offe, Claus. 2009. Governance: An „Empty Signifier“? Constellations 16 (4): 550-562.

OSZE, Hrsg. 2003. The impact of media concentration on professional journalism. Wien: Office of the OSCE Representative on Freedom of the Media.

Padovani, Cinzia. 2007. A fatal attraction. Public television and politics in Italy. Lanham: Rowman and Littlefield Publishers.

Puppis, Manuel. 2010. Media governance: A new concept for the analysis of media policy and regulation. Communication, Culture \& Critique 3: 134-149.

Reh, Christine. 2012. Informal politics: The normative challenge. In International Handbook on Informal Governance, Hrsg. Thomas Christiansen und Christine Neuhold, 65-84. Cheltenham: Edward Elgar.

Reporter ohne Grenzen. 2013. Wolrd report. http://en.rsf.org/report-mexico,184.html. Zugegriffen: 06. Mai 2014.

Schlichte, Klaus. 2012. Der Streit der Legitimitäten. Der Konflikt als Grund einer historischen Soziologie des Politischen. Zeitschrift für Friedens- und Konflikforschung 1 (1): 9-43.

Schudson, Michael. 2006. The troubling equivalence of citizen and consumer. In The Annals of the American Academy of Political and Social Science 608: 193-204. 
SGI network. 2011. Italy country report. http://www.sgi-network.org/pdf/SGI11_Italy.pdf. Zugegriffen: 06. Mai 2014.

Stacey, Jeffrey. 2010. Integrating Europe: Informal politics and institutional change. Oxford: Oxford University Press.

Strömbäck, Jesper. 2008. Four phases of mediatization: An analysis of the mediatization of politics. The Harvard International Journal of Press/Politics 13 (3): 228-246.

Terzis, Georgios. 2007. European media governance: National and regional dimensions. Chicago: Intellect Books, The University of Chicago Press.

Tranquina, Nelson. 1995. Portuguese television: The politics of savage deregulation. Media, Culture and Society 17: 223-238.

Trappel, Josef, Werner A. Meier, Klaus Schrape und Michaela Wölk. 2002. Die gesellschaftlichen Folgen der Medienkonzentration. Veränderungen in den demokratischen und kulturellen Grundlagen der Gesellschaft. Opladen: Leske u. Budrich. 